\title{
Quality Management Systems within the Public Sector: The Case of ISO 9000 Implementation Barriers in Malaysian Local Government
}

\author{
Shardy Abdullah ${ }^{1}$, Arman Abdul Razak ${ }^{2}$, Mohd Hanizun Hanafi ${ }^{3,}$ \\ Nor'Aini Yusof ${ }^{4}$ \\ ${ }^{1234}$ School of Housing, Building and Planning, Universiti Sains Malaysia, Pulau Pinang, Malaysia
}

\begin{abstract}
Implementing ISO 9000 in the public sector is normally seen to be wrought with various obstacles that usually impede the entire process. The objective of this study is to explore the main barriers of ISO 9000 implementation in Malaysian Local Government $(L G)$ organizations. Relevant information had been gathered from literature in developing the survey tool to gather the related data. Quantitative analysis was then used to analyse the data. There were three aspects discovered to be the main barriers which have hampered the ISO 9000 implementation process. These barriers are lack of cooperation among internal departments, negative perception or attitude towards quality among employees and employees' culture toward quality.
\end{abstract}

Keywords- Quality management systems, public sector, ISO 9000, implementation barriers, local government

\section{Introduction}

As a government agency, $\mathrm{LG}$ administration has been a constant subject of administrative reforms in Malaysia [1]. Therefore, LG is responsible to follow and implement any improvement programme that is introduced and instructed by the Federal Government including the instruction for implementing ISO 9000 within its organisation. The directive to implement this quality management system was spelt out in the Civil Administration Improvement Circular, no. 2 year 1996 where through this circular the government has instructed all its agencies, including LGs to implement ISO 9000. Subsequently, in 2002, the government had once again issued another Civil Administration Improvement Circular, no. 2 year 2002, where this new circular announced the cancellation of the use of the ISO 9000:1994 standards and superseded it with the ISO 9000:2000 version in line with the changes made by the governing International ISO body. However, since ISO 9000:2000 was then updated to the 2008 version, the government once again instructed its agencies, including LG organizations to update the ISO implementation according to the latest version. This directive was stated in the MS ISO 9001:2008 Implementation Guidelines for the Public Sector, dated January $1^{\text {st }}$, 2010. To make the implementation of the quality management system simpler and consequently scheduling the accreditation and certification process for ISO 9000 within a reasonable timeframe for every agency involved, the circular came attached with a precise and detailed implementation guideline. In addition to this, the Malaysian Administrative Modernisation and Management Planning Unit (MAMPU) was specifically appointed by the government to guide and advice the LG organizations in the implementation process.

The implementation of the ISO 9000 also complements and completes the efforts to upgrade quality which have been and are currently undertaken by government agencies, where, through the advent of ISO 9000, quality management has become even more prominent via the pursuit of Total Quality Management (TQM) programmes for organisations to improve competitiveness [2]. Within the LG context, the implementation of ISO 9000 will enable these organisations to benefit from other advantages such as a continuous process of quality improvement [3], as well as contributing greater awareness of customer needs and improved customer relationships [4], savings in costs, time and effort [5] and other administrative and managerial benefits. Nevertheless, after a decade since ISO 9000 was introduced by the government the number of LG organizations which have obtained the ISO 9000 (latest version) accreditation was very limited. If looked into in general, this situation should have never occurred since it involved a relatively long implementation timeframe, approximately 14 years (1996-2010) and the government had also provided a proper implementation guideline as well as appointing MAMPU as an advisory and consulting body to be fully utilized by the LGs in implementing ISO 9000. This study aims to discover the main barriers faced by Malaysian LGs in implementing the ISO 9000 quality system.

\section{The ISO 9000 Implementation Barriers}

Previous literature in general describes a variety of barriers associated with the implementation of ISO 9000 for either 1994, 2000 or 2008 versions. Wahid and Corner [6], in a study about the critical success factors and problems in ISO 9000 maintenance have stated that one of the main barriers is largely due to a lack of top 
management involvement and understanding of ISO 9000 requirements for the quality systems of the companies. This is congruent with the discovery by Magd [7] who explained that the most crucial barrier was perceived as being top management commitment and this is due to the fact that without top management commitment, employees tend to put a low priority on the quality management system and as a result, the quality management system becomes static. In addition, commitment from the top management has been identified as one of the critical success factors that directly influence the success of implementing ISO 9000 within any organization.

Furthermore, based on a research by Bhuiyan and Alam [8], it was discovered that initially there were 12 statements listed as implementation barriers, but in the end, only 4 from those barriers were found to be the actual barriers. These barriers are constraints on resources, underestimation of efforts needed for registration, implementation and documentation of established procedures document development as well as high preparation cost. Through this discovery, it can been seen that the need for resources, perception, development of documentation and the cost factor are able to impede the ISO 9000 implementation process within an organization. Implementing ISO 9000 does certainly require the involvement of several resources, especially human and financial resources. An adequate financial resource is needed by an organization to offset the related costs incurred throughout the ISO 9000 implementation process, such as fees for consultancy services, training fees, costs related to certification processes as well as other relevant costs. For some organizations, these costs and fees may be deemed as relatively high and may also become a burden for them. On the other hand, human resources are also necessary to execute activities concerning documentation and internal audits. In an organization with a relatively small number of employees, ISO 9000 implementation can be seen as introducing additional work loads onto the employees, especially in the aspect of documentation activities.

Besides that, Bhuiyan and Alam [9] in their study on ISO 9000 implementation in small manufacturing firms have also identified quality perception and resistance to change as part of the barriers which hamper the quality system implementation process. When these two barriers are further scrutinized, it can be clearly seen that they are directly connected to human behaviour or attitude. If the personnel or employees responsible for ISO 9000 implementation themselves possess a negative perception towards the quality management system, it would be only logical that their eagerness in achieving ISO 9000 certification would also be significantly less. Subsequently, without these concerted serious efforts, the implementation process would also suffer from delays or even worse, halted indefinitely. On the other hand, resistance to change can also be deemed as a barrier as the very essence of ISO 9000 implementation is aimed at bringing change within the level of service provided by an organization. This resistance to change is often brought on by the perceived contentedness and envisioned comfort zone by certain parties towards their respective achievements. In an earlier study, Amar and Mohd Zain [10] had listed several barriers faced during ISO 9001:2000 implementation where those barriers were categorized into a few sections and sub-sections such as quality support, internal communication support, resource quality, competent personnel, competence support, infrastructure quality and process control. However, the most frequent barrier according to the opinion of the respondents pertains to the human resource factor such as insufficient education level of workers, low worker morale, lack of skill among workers, high employee turnover and indiscipline. Apart from these main barriers are discussed above, through a number of research conducted on the difficulties faced by companies that embark on the ISO implementation process, several hindrances have been determined, such as for example, lack of training resources or understanding of the requirements and benefits of being ISO certified [11], time consuming, difficulties in interpreting the standards, unclear guidelines from the certifying body [12], documentation control [13], the type of power in organizations, the distribution of authority, the degree of empowerment, organizational politics, leadership style and conflicts within the organization [14] as well as other obstacles.

In addition to these stated barriers, through a study conducted on the implementation of quality programmes in Malaysian libraries, Osman et al. [15] divided the obstacles concerned into three main groups. The first are barriers relating to problems involving incompetent personnel, apathy, no commitment, lack of staff, untrained workers and other similar problems. The next group of barriers dealt with insufficient time due to staff being committed to administrative work and tasks, implementation of quality being not perceived as core activities, the incidences of ad-hoc programmes and other related problems. The final group of barriers was closely tied in with the problem of insufficient financial allocation. Besides this, Yaacob [16] in discussing issues related to the performance of local government bodies from the perspective of implementing quality management programmes within their organizations, concluded that there are various factors that contribute towards the existence of implementation barriers. These factors hinge largely on three main dimensions, which are imitated quality management practices, tenure of quality management implementation and a limited organizational focus on only particular aspects of quality management.

By reviewing all these discussions, it is discovered that the barriers faced by both public and private organizations in Malaysia in implementing ISO 9000 are very much identical to the problems faced elsewhere in the world. As a summary, these implementation barriers could be connected to four themes which are related to 
organizational, resource, human and technical aspects. From the organizational aspect, the barriers include any weakness or shortcomings related to administrative affairs, management, development, structure, focus and work culture that have occurred or are being practiced in an organization such as lack of top management support and commitment, lack of training and education of employees, difficulty in allocation of responsibilities and authority of personnel, lack of necessary guidance for certification, lack of cooperation among internal departments, lack of leadership, underestimation of efforts needed for registration, lack of implementation planning, lack of recognition or rewards system, lack of communication and others. From the resource aspect on the other hand, the barriers are related to lack or absence of resources that are needed in implementing ISO 9000. These resources comprise of financial, equipment, staff, information, time as well as other similar aspects. Meanwhile, the barriers related to the human aspect consist of attitude weaknesses, comprehension and perception, practices, readiness and culture of certain parties that are involved with the ISO 9000 implementation process. Among the barriers are resistance to change, unclear benefits of obtaining certification, lack of understanding on the importance of ISO 9000, workload increase, employees' culture towards quality, negative perception or attitude towards quality, attitude of third parties such as consumers or suppliers, problems relating to roles or attitudes of quality assessors or consultants, lack of involvement, cooperation and commitment from employees and lack of motivation. Finally, the barriers that are associated with the technical aspect are the inability to undertake or cater some requirements fixed by the ISO 9000 quality management system itself. There are many barriers involved such as lack of understanding of the ISO 9000 system, difficulty in interpreting ISO 9000 requirements, difficulty in fulfilling ISO clauses and requirements, improper control of documents and data difficulty in development of documentation and difficulty in implementing the quality audit process.

\section{Methodology}

The data was collected through questionnaires distributed to respondents consisting of quality managers/heads or quality members in LG organizations within Peninsular Malaysia. At present, there are 99 LG organizations in Peninsular Malaysia. However, only 53 LGs agreed to participate in this research as the corresponding survey respondents. The development of the questionnaire content for this study was primarily based on the information that had been gathered from secondary resources especially from journal articles. As the sample size of the data is small (only involving 53 respondents), according to Hair et al. [17], it is not suitable for validity tests such as exploratory factor analysis (recommended sample size: 100) or confirmatory factor analysis (recommended sample size: 150). As such, in this study, a reliability test using the Cronbach Alpha coefficient was undertaken in order to determine the consistency of the measures. Typically, the higher values of Cronbach's alpha indicate a higher degree of reliability.

\subsection{Reliability Result}

\section{Results And Discussion}

It was revealed that the values of Cronbach's alpha for this study range from 0.752 to 0.835 . According to Nunnaly [18] and Litwin [19], the reliability value of 0.7 or higher is considered to be adequate. Therefore, as all the recorded values of Cronbach's alpha for each of the respective barrier categories exceeded 0.7, it can be subjectively concluded that the survey instrument utilized in this research is reliable and internally consistent.

\subsection{The Barriers}

The garnered data was then subsequently analyzed based on the respective barrier categories using the descriptive statistics approach. Table 1 shows the results of this analysis.

Table 1. Results of descriptive analysis based on barriers

\begin{tabular}{|l|l|l|l|l|}
\hline No. & List of Barriers & Mean & Mode & SD \\
\hline 1. & Lack of cooperation among internal departments & 4.06 & 4.00 & 0.91 \\
\hline 2. & Increase of workload & 3.08 & 2.00 & 1.16 \\
\hline 3. & Lack of top management support and commitment & 2.91 & 2.00 & 1.38 \\
\hline 4. & Lack of training and education of employees & 2.64 & 2.00 & 1.21 \\
\hline 5. & Lack of leadership & 2.13 & 2.00 & 0.73 \\
\hline 6. & Lack of implementation planning & 2.04 & 2.00 & 1.09 \\
\hline 7. & Difficulty in allocation of personnel responsibilities and authority & 2.02 & 2.00 & 0.89 \\
\hline 8. & Underestimation of efforts needed for registration & 1.96 & 2.00 & 0.76 \\
\hline 9. & Lack of recognition or rewards system & 1.80 & 1.00 & 0.86 \\
\hline 10. & Lack of necessary guidance for certification & 1.75 & 1.00 & 0.88 \\
\hline 11. & Lack of communication & 1.74 & 1.00 & 0.90 \\
\hline
\end{tabular}


Quality Management Systems Within The Public Sector: The Case Of ISO 9000 Implementation

\begin{tabular}{|l|l|l|l|l|}
\hline 12. & Lack of human resources & 3.91 & 4.00 & 1.16 \\
\hline 13. & Lack of time & 2.94 & 2.00 & 1.13 \\
\hline 14. & Lack of financial resources & 2.85 & 2.00 & 1.08 \\
\hline 15. & Lack of qualified personnel & 2.62 & 2.00 & 1.02 \\
\hline 16. & Lack of related information & 2.34 & 2.00 & 0.79 \\
\hline 17. & Negative perception or attitude towards quality among employees & 4.17 & 4.00 & 0.89 \\
\hline 18. & Employees' culture toward quality & 4.06 & 5.00 & 1.08 \\
\hline 19. & Lack of involvement, cooperation and commitment from employees & 3.94 & 4.00 & 0.99 \\
\hline 20. & Lack of understanding on the importance of ISO 9000 & 2.70 & 2.00 & 0.99 \\
\hline 21. & Unclear benefits of obtaining certification & 2.70 & 2.00 & 0.91 \\
\hline 22. & Lack of motivation & 2.62 & 2.00 & 1.02 \\
\hline 23. & Negative attitude to quality among customers and suppliers & 2.60 & 2.00 & 0.84 \\
\hline 24. & Resistance to change & 2.53 & 2.00 & 0.85 \\
\hline 25 & $\begin{array}{l}\text { Problems regarding the role and attitude of quality auditors and } \\
\text { consultants }\end{array}$ & 2.36 & 2.00 & 0.60 \\
\hline 26. & Lack of understanding of the ISO 9000 system & 2.41 & 2.00 & 1.12 \\
\hline 27. & Difficulty in implementing quality audit process & 2.23 & 2.00 & 0.72 \\
\hline 28. & Difficulty in fulfilling the ISO clauses and requirements & 2.21 & 2.00 & 0.88 \\
\hline 29. & Difficulty in interpreting ISO 9000 requirements & 2.04 & 2.00 & 0.76 \\
\hline 30. & Difficulty in development of documentation & 1.92 & 2.00 & 0.87 \\
\hline 31. & Improper control of documents and data & 1.70 & 2.00 & 0.64 \\
\hline 32 & Problems related to instruments, equipment and tools. & 1.60 & 2.00 & 0.57 \\
\hline
\end{tabular}

*Note: Scale $1=$ strongly disagree, $2=$ disagree, $3=$ neutral, $4=$ agree and 5 strongly agree

Based on the above table, it was discovered that from the total of 32 barriers listed, there were only 3 barriers that recorded a mean and mode score exceeding 4.0. These three barriers are lack of cooperation among internal departments from the organizational aspect and another 2 barriers categorized under the human aspect, namely negative perception or attitude towards quality among employees, and employees' culture toward quality. This analysis has directly revealed that there are only 3 main barriers which have truly hindered or obstructed the ISO implementation process in most LG organizations. Referring to the first main barrier, which is lack of cooperation among internal departments, the existence of this barrier in hampering the ISO 9000 implementation process has somewhat been expected. This is because LG organizations normally have an organizational structure that is made up of several sub-departments that have their own responsibilities and roles. The establishment of these departments are inline with the diverse and far reaching roles as well as functions that a LG organization plays. The diversity of these departments is actually to ensure that a LG organization is capable in providing all the necessary services in a systematic and effective manner. However, in the context of ISO 9000 implementation, the existence of these numerous departments have made it difficult for the LG organization to implement ISO 9000 as the level of cooperation accorded is different from one department to another. As Chu et al. [20] state, the existence of a good and consistent internal cooperation is a critically required factor in ensuring the success of ISO 9000 implementation within a public sector organization. There are various reasons that contribute to this barrier, among others being lack of commitment and focus from heads of department [21], a low level of awareness and understanding towards the importance of ISO 9000 implementation [22], weak internal communication [23] and insufficient leadership shown by heads of department.

The other two barriers, namely, negative perception or attitude towards quality among employees and employees' culture toward quality, are also barriers that frequently exist in ISO 9000 implementation, as previously identified by Mo and Chan [24], [10] and Yahya [25]. It is a general notion that workers are considered to be the most important asset for any given organization, including LG agencies. This is due to the fact that workers are the main drivers in executing each and every activity or program that has been outlined for implementation within an organization. This also rings true in ISO 9000 implementation for LG organizations, where the positive and committed attitude and contributions of the workers are key in ensuring every process in done correctly, in a timely fashion and reaches its intended aim. When there is negative attitude among workers towards ISO 9000 implementation, employees would begin to feel that they are controlled by the system, hence they will be reluctant to adopt the change. A study by Koo et al. [26] on the attitude of employees towards ISO 9000 implementation has revealed that employees felt the decline of quality success drivers in the midst of the ISO journey as compared to the start. As such, a consistent and continuous positive attitude and motivation in employees is required to ensure that they are continually driven to implement the ISO 9000 process until 
certification is achieved and subsequently, motivated enough to maintain and sustain the acquired accreditation. This is congruent with the findings of Yung [27] who stressed that the participation of all employees at every level is needed in making the ISO 9000 implementation process a success in an organization.

Besides these 3 discussed barriers, there are 2 other barriers; lack of human resources related to the resource aspect; and lack of involvement, cooperation and commitment from employees under the human aspect which returned a mean score close to 4.0 and at the same time recorded a high mode value of 4.0. Based on these scores, these two barriers should be considered as part of the main barriers that influence the effective implementation of the ISO 9000 process in LG organizations. The existence of this lack of human resource barrier is a challenge of sorts to most LG organizations since they are statutory bodies that need to get prior approval from the Malaysian Public Service Department before being able to offer new positions in their respective organizations. Coupled with the financial constraints that are already part of the problems faced by most LG organizations, the ability of a LG organization in procuring or hiring more employees is seen to be rather limited. The barrier regarding lack of involvement, cooperation and commitment from employees can be regarded as a consequent issue that occurs due to two pre-existing barriers of negative perception or attitude towards quality among employees as well as employees' culture toward quality. This is because these two barriers will create negative action and discouragement among the employees to be fully involved in the ISO implementation process within their respective organizations. When these employees possess adverse perception or attitude, those who are involved in ISO implementation will adopt a lackadaisical mindset and this in turn will cause their level of participation to become restricted leading to reluctance in terms of cooperation as well as lack of full undivided commitment. Based on these findings, it can be deduced that the two main barriers usually present in the ISO 9000 implementation process, namely lack of top management support and commitment as well as lack of training and education of employees as highlighted by a majority of previous studies do not in actual fact hinder the implementation of ISO 9000 within LG organizations in Malaysia.

Apart from the 32 barriers as listed previously, this research had also found 3 other additional barriers, namely, change of Council President/Mayor; no proper quality management department, division or unit; and change of ISO 9000 version. Nonetheless, the existence of these three barriers needs to be given due consideration and focus so that these issues would not be prolonged to ultimately become one of the main barriers for LG organizations to implement ISO 9000 quality management systems in their organizations. The issue of change in council presidents or mayors is certainly an aspect that will negatively influence ISO 9000 implementation in LG organizations. This change is top management usually occurs within a very short time cycle, around every 2 to 5 years. This scenario will stunt the ISO 9000 implementation process in LG organizations as every officer elected as Council President or Mayor will have a different and varying interest, awareness and priority towards ISO 9000 implementation in the organizations that they lead. In addition to this, the absence of a proper department, section or unit in handling quality management aspects can be regarded as a form of hindrance for ISO 9000 implementation in LG organizations as there would not be any specifically appointed employees to oversee and manage this implementation process. Without specific employees, the task and responsibility of ISO 9000 implementation would normally be assigned to other employees as a form of additional duties when in fact those employees are already obligated to perform their core activities. In a situation such as this, these employees will have a tendency to execute the ISO 9000 processes in a apathetic manner. On the other hand, changes in the ISO 9000 version can also be considered as a complication in implementing ISO 9000 within LG organizations. The change of versions will undoubtedly cause the requirements for ISO 9000 certification to change accordingly, and this in turn will necessitate the employees involved in a certain implementation process to modify and revise their activities to suit the new requirements.

\section{Conclusion}

The efforts of the government to ensure LG organizations implement and consequently achieve ISO 9000 accreditation has indeed been clearly stated through a government circular issued in 1996. In fact, after the change of the ISO 9000 version, in 2002 and 2010 respectively, the government still showed their stand in enforcing LG organizations to implement ISO 9000 within their organizations according to the latest version. Although the intent and the implementation directive have been explicitly stated by the government, the number of LG organizations that have obtained ISO certification is still small and far from the target set by the government. Through the survey done within this study, it was discovered that there are 3 main barriers which have hampered and hindered the implementation of ISO 9000, resulting in a limited number of LG organizations that have succeeded in achieving this quality system accreditation. These findings are somewhat parallel to the categories of barriers that have been highlighted by previous studies. However, the findings of this study show that the main barriers in the process of implementing ISO 9000 within LG organizations are not completely similar to the main barriers that have been generally identified previously. From the total of 3 identified barriers, it was discovered that these barriers are related to the organization, resource as well as human aspect. This study also discovered several new barriers that disrupt the ISO 9000 implementation process in LG organizations 
where they pertain to the administrative practices adopted by the organizations as well as changes within the ISO 9000 version itself. Based on this finding, the responsible parties should adapt specific initiatives and approaches to mitigate these identified problems. Continuous disregard will cause this situation to become more serious and subsequently the aim of the government in ensuring its agencies implement ISO 9000 quality management systems will merely become an unachieved aspiration. To avoid this issue from becoming a reality, it is appropriate that further research be done on the ISO 9000 implementation processes within LG organizations, specifically to review the necessary actions and strategies that should be put into place to prevent, minimize or eradicate the problems faced by the LG ISO 9000 implementation team. This is to ensure that the implementation process of the quality management system can be conducted in a systematic, smooth and effective manner.

\section{Acknowledgement}

The authors would like to express their thanks to Universiti Sains Malaysia as this research was sponsored under the Universiti Sains Malaysia Short Term Grant, account no.: 304/PPBGN/638169, entitled 'An Investigation on MS ISO 9000 Implementation Process in Local Authority Organization'.

\section{References}

[1] Salleh, D. and Abdul Khalid, S.N. (2011). Accountability Practice at Local Government of Malaysia. Proceeding of 2nd International Conference On Business And Economic Research (2nd ICBER 2011), 1305-1314.

[2] Kuo, T., Chang, T.J., Hung, K.C. and Lin, M. Y. (2009). Employees' perspective on the effectiveness of ISO 9000 certification: A Total Quality Management framework. Total Quality Management \& Business Excellence, 20 (12), 1321 - 1335.

[3] Mohd. Yusoff Zakaria (1997), Penambahbaikan Kualiti yang Berterusan Melalui MS ISO 9000, Proceeding of Simposium Kebangsaaan MS ISO 9000 on 6-7 January 1997, Kuala Lumpur, Malaysia.

[4] Narayana, P. and Goudar, I.R.N. (1999). Implementation of ISO 90000: A Case Study of NAL Information Centre, presented in DRTC Workshop on Information Management at 6-7 January 1999, Bangalore, India.

[5] Halim Shafie and Manogran, P. (1999), MS ISO 9000: A Practical Guide for Implementation in the Civil Service. Kuala Lumpur, National Institute of Public Administration.

[6] Wahid, R. A. and Corner, J. (2009). Critical success factors and problems in ISO 9000 maintenance. International Journal of Quality \& Reliability Management, 26(9), $881-893$.

[7] Magd, H.A.E (2008). ISO 9001:2000 in the Egyptian manufacturing sector: perceptions and perspectives. International Journal of Quality \& Reliability Management, 25(2), $173-200$.

[8] Bhuiyan, Nadia and Alam, Nadeem (2005b). An investigation into issues related to the latest version of ISO 9000. Total Quality Management \& Business Excellence, 16(2), 199 - 213.

[9] Bhuiyan, N. and Alam, N. (2005) A case study of a quality system implementation in a small manufacturing firm. International Journal of Productivity and Performance Management, 54(3), 172-186.

[10] Amar, K. and Mohd Zain, Z. (2002). Barriers to implementing TQM in Indonesian manufacturing organizations. The TQM Magazine, 14(6), $367-372$.

[11] Chin, K. W., Poon, G. K. K. \& Pun, K. F. (2000) The critical maintenance issues of the ISO 9000 system: Hong Kong manufacturing industries' perspective. Work Study, 49, 89-96.

[12] Carlsson, M. and Carlsson, D. (1996). Experiences of implementing ISO 9000 in Swedish industry. International Journal of Quality \& Reliability Management, 13(7), $36-47$.

[13] Erel, E. and Ghosh, J.B. (1997). ISO 9000 implementation in Turkish industry. International Journal of Operations \& Production Management, 17(12), 1233 - 1246

[14] Low, S.P. and Chan, F.M. (1998) Quality management systems: a study of authority and empowerment. Building Research and Information, 25(3), 158-169.

[15] Osman, Z., Goon, C. A., Aris, W. H. W. (1998). Quality services: Policies and Practices in Malaysia. Library Management, 19(7), $426-433$.

[16] Yaacob, Z. (2010). Quality management as an effective strategy of cost savings African Journal of Business Management Vol. 4(9), 1844-1855.

[17] Hair, J.F., Black, W.C., Babin, B.J., Anderson, R.E., and Tatham, R.L. (2006). Multivariate data analysis 6th Edition. Pearson Prentice Hall. New Jersey.

[18] Nunnaly, J. (1978). Psychometric theory. New York: McGraw-Hill.

[19] Litwin, M. (1995). How to Measure Survey Reliability and Validity. Survey Kit. Vol. 7. A. Fink. Beverly Hills, CA, Sage Publications.

[20] Chu, P., Huang, C., and Wang, H. (2001). ISO 9000 and Public Organizations in Taiwan: Organizational Differences in Implementation Practices with Organization Size, Unionization and Service Types. Public Organization Review, 1(4), 391-413.

[21] Schuurman, H. (1997). Quality Management and Competitiveness-The Diffusion Of The ISO 9000 Standards In Latin America And Recommendations For Government Strategies. Santiago, Chile: Division of Production, Productivity and Management, UNITED NATIONS.

[22] Al-Zamany,Y., Hoddell, S.E.J. and Savage, B.M., (2002). Understanding the difficulties of implementing quality management in Yemen. The TQM Magazine, 14(4), $240-247$.

[23] Vloeberghs, D. (1996). ISO 9000 in Belgium: Experience of Belgian quality managers and HRM. European Management Journal, 14(2), 207-211.

[24] Mo, J. and Chan, A. (1997). Strategy for the successful implementation of ISO 9000 in small and medium manufacturers. The TQM Magazine, 9(2), 135-145.

[25] Yahya, S. (2003). The Role of ISO 9001: 2000 Consultants. IIUM Journal of Economics and Management. 11(2), 143-165.

[26] Koo, H., Koo, L.C. and Tao, F. K.C. (1998). Analysing employee attitudes towards ISO certification. Managing Service Quality, 8(5), 312-319.

[27] Yung, W.K.C. (1997). The values of TQM in the revised ISO 9000 quality system, International Journal of Operations \& Production Management, 17(2), 221-230. 